\title{
Contenido
}

Presentación

Editorial.

Viviana Cervantes Atia

Pabla Peralta Miranda

Universidad Simón Bolívar, (Barranquilla - Colombia)

Los factores que conforman el modelo de acreditación por alta calidad de programas académicos en Colombia, Revisión desde lenfoque la percepción.......................................................

The factors that constitute the model of accreditation for high quality of academic programs in Colombia, review from the approach of perception

Adriana Patricia Uribe Uràn

Universidad Simón Bolívar, (Barranquilla - Colombia)

Uso de las tic en la gestión del conocimiento en la Universidad..... 55-93 del Zulia (núcleo col)

Use of the ict in knowledge management at Universidad de Zulia (Nucleo col)

Josmary del Valle Rojas Oriquen

Dubisay Thais Morales Gutiérrez

Carlos Antonio. García Mora

Universidad del Zulia, (Venezuela)

Satisfacción laboral y el clima organizacional de las principales universidades sucreñas

Satisfaction and organizacional climate of the main universities in Sucre

Claudia Patricia Rojas Martínez

Corporación Universitaria del Caribe CECAR, (Sucre- Colombia) 
Identificación de factores no monetarios asociados a las pymes del sector confecciones a mercados internacionales.................

Identification of factor sassociated with nomonetary sector Pymes affect your income sized international market

Carlos de Jesús Fábregas Rodado

Universidad Simón Bolívar, (Barranquilla- Colombia)

\section{La asociatividad como estrategia para mejorar la competitividad de las microempresas productoras de calzado del municipio} de Sincelejo

the associativity as a strategy to improve the competitiveness of producing msmes shoe township Sincelejo

Luis Isaac Esquivia Salgado

Universidad de Sucre, (Colombia)

\section{Estrategias utilizadas por las empresas constructoras en Sincelejo para tener ventajas competitivas.}

Strategies used by the construction companies in competitive advantages to have Sincelejo

Alfredo Carlos Yemail Barragán Universidad Corporación Universitaria del Caribe CECAR, (Sucre - Colombia) Atlantis Constructora 
Consideraciones teóricas de estrategia y gestión en el

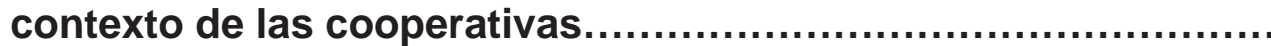

Theoretical considerations strategy and management in the context of the cooperative

Madelin Sánchez Otero

Luisa Sánchez Otero

Corporación Universitaria de la Costa CUC

Proyecto Eco turístico, en la región Caribe, colombiana................

Ecotourism project in the Colombian Caribbean region

Enna Lucia Sarmiento Estrada

Cristian Alberto Correa Garrido,

Universidad Simón Bolívar, (Barranquilla, Colombia) 